\title{
Discrepancy between Subcritical and Fast Rupture Roughness: A Cumulant Analysis
}

\author{
N. Mallick, P.-P. Cortet, S. Santucci, ${ }^{*}$ S. G. Roux, and L. Vanel ${ }^{\dagger}$ \\ Laboratoire de physique, CNRS UMR 5672, Ecole Normale Supérieure de Lyon, 46 allée d'Italie, 69364 Lyon Cedex 07, France
}

(Received 28 July 2006; published 20 June 2007)

\begin{abstract}
Rough crack fronts in a sheet of paper, obtained during a creep experiment, do not follow true scaling laws. Local roughness exponents are estimated using the first order cumulant, a quantity recently introduced in the turbulence literature [J. Delour, J. F. Muzy, and A. Arneodo, Eur. Phys. J. B 23, 243 (2001)]. Using a large data set (102 fronts), we find a significant difference in local roughness between the slow (subcritical) and the fast growth regime.
\end{abstract}

DOI: $10.1103 /$ PhysRevLett.98.255502

PACS numbers: 62.20.Mk, 02.50. $-\mathrm{r}, 68.35 . \mathrm{Ct}$

Since the early description of rough fractures as selfaffine surfaces [1], the existence of universal roughness exponents has been strongly debated [2]. There are now many experimental evidences for a nonuniversal value of the roughness exponent of fracture surfaces. Different exponents can be found due to the anisotropy of the fracturation process [3], the heterogenity of the material structure [4], or anomalous scaling related to finite-size effects [5]. A recent observation suggests that, in rupture of paper, the crack interface would be multifractal [6].

Roughness exponents appear usually independent of crack velocity. For rather slow velocities, no effect of the velocity on roughness has been observed in Plexiglas $\left(v=10^{-7}-5 \times 10^{-5} \mathrm{~m} \mathrm{~s}^{-1}\right)$, glass $\left(v=10^{-9}-5 \times\right.$ $\left.10^{-8} \mathrm{~m} \mathrm{~s}^{-1}\right)$, intermetallic alloys $\left(v=10^{-8}-5 \times\right.$ $\left.10^{-5} \mathrm{~m} \mathrm{~s}^{-1}\right)$, or sandstone $\left(v=10^{-4}-10^{-2} \mathrm{~m} \mathrm{~s}^{-1}\right)$ [7]. There is also no effects of applied strain rate on crack roughness in paper [8]. On the contrary, in dynamic fracture of Plexiglas $\left(v \geq 600 \mathrm{~m} \mathrm{~s}^{-1} \simeq 0.45 \mathrm{x}\right.$ Rayleigh wave speed), variations of the roughness exponents with the velocity have been observed [9]. As pointed out in a recent review [10], there is a need for experimental studies concerning the influence of fracture kinetics on roughening.

In this Letter, we study the roughness of a crack interface in a sheet of paper [11] fractured in a creep experiment [12]. During each experiment, crack growth starts in a subcritical regime where the growth is slow $(v=$ $10^{-5}-10^{-2} \mathrm{~m} \mathrm{~s}^{-1}$ ) and reaches at a critical length a fast growth regime $\left(v \sim 300 \mathrm{~m} \mathrm{~s}^{-1}\right)$. We compare the scaling properties of moments [13] to the scaling properties of cumulants, a new quantity recently introduced in the turbulence literature [14]. We find that there is a systematic difference between the scaling properties of the subcritical and the fast rupture regime. In contrast to moments, the first cumulant is insensitive to deviations from monofractal behavior and allows us to extract reliably a roughness exponent.

Experiments. -We recall briefly the experimental setup described in [12]. We break bidimensional brittle samples made of fax paper sheets (Alrey) with size $24 \times 21 \mathrm{~cm}^{2}$. Each sample has an initial centered crack and is loaded in a tensile machine with a constant force $F$ perpendicular to the crack direction (mode I). The stress intensity factor $K(L) \propto F \sqrt{L}$, where $L$ is the crack length, determines the stress magnitude near the crack tip and is the control parameter of crack growth. For a given initial length $L_{i}$, subcritical crack growth is obtained by choosing $F$ so that $K\left(L_{i}\right)$ is smaller than a critical threshold $K_{c}$, corresponding to the material toughness. During an experiment, $L$ increases, and so does $K(L)$. It will make the crack accelerate until reaching the critical length $L_{c}$ for which $K\left(L_{c}\right)=$ $K_{c}$ and above which a sudden transition to fast crack propagation occurs. Using a high speed camera (Photron Ultima 1024), we have determined which part of the post mortem crack interface corresponds to slow or fast growth, and measure the velocity of the crack in each one. In the subcritical regime, the velocity ranges from $10^{-5}-10^{-2} \mathrm{~m} \mathrm{~s}^{-1}$. Recording at $4000 \mathrm{fps}$, we find a crack velocity about $300 \mathrm{~m} \mathrm{~s}^{-1}$ in the fast regime. Note that there are four to seven decades between the two growth regime velocities.

Crack profiles. - Post mortem samples are digitized with a scanner at $1600 \mathrm{dpi}$. The pixel size $a_{0}=16 \mu \mathrm{m}$ is close to the typical diameter of cellulose fibers. In Fig. 1, we show an example of a digitized sample compared with the extracted crack front $s(x)$. We distinguish between different stages corresponding to: $(a)$ the initial crack, $(b)$ the subcritical crack growth, and (c) the fast crack growth. We have digitized 51 fractured samples, obtained for different forces $(200 \mathrm{~N}, 230 \mathrm{~N}, 250 \mathrm{~N}, 280 \mathrm{~N})$ and initial crack sizes $(1,2 \mathrm{~cm})$. Since the initial crack is centered, each sample give rise to two fronts. Thus, we have a total of 102 independent fronts of about $10^{3}$ points for slow growth and $10^{4}$ points for fast growth.

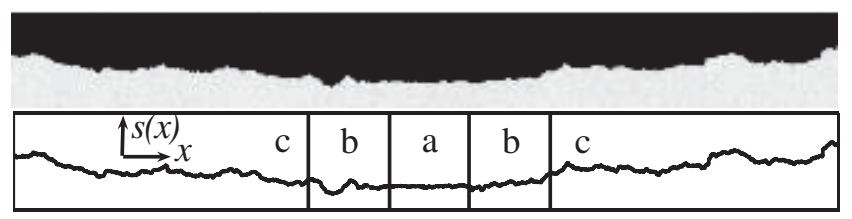

FIG. 1. Digitized post mortem sample and corresponding extracted front showing three stages: (a) initial crack $\left(L_{i}=2 \mathrm{~cm}\right)$, (b) subcritical growth, (c) fast growth. 
Scale invariance. - Let $\{s(x), x \in \mathbb{R}\}$ be a signal as a function of a coordinate $x$. Scale invariance of $s$ means that there is no characteristic scales in the signal. The scaling properties of $s$ can be characterized by introducing a multiresolution coefficient $T[s](x, a)$ defined at scale $a$ and position $x$. Scale invariance implies that the $q$ th order moments of the multiresolution coefficient follow a power law with exponents $\zeta(q)$ :

$$
M_{q} \equiv\left\langle|T[s](x, a)|^{q}\right\rangle \sim C a^{\zeta(q)},
$$

where the bracket denotes the average over the $x$ space. The increments over a scale $a, T[s](x, a)=s(x+a)-$ $s(x)$, are standard multiresolution coefficients and the corresponding moments are the structure functions [15]. It has been shown that a more general framework for defining multiresolution coefficients is the wavelet transform [16$18]$. When the signal $s$ follows Eq. (1) with $\zeta(q)$ proportional to $q$, the signal is monofractal. The complete analysis of the deviations of $\zeta(q)$ from monofractality can be made through the multifractal formalism.

Multifractal analysis. - Multifractal formalism is based on the mathematical definition of a local singularity exponent $h(x)$. Using the multiresolution coefficient $T[s] \times$ $(x, a)$, we write at each position $x$ [17]

$$
|T[s](x, a)| \sim C a^{h(x)},
$$

where $h(x)$ is the Hölder exponent or local roughness exponent describing how singular the signal is at position $x$ : the larger $h(x)$, the smoother $s(x)$. The statistical distribution of the Hölder exponents is quantified by the singularity spectrum $D(h)$ defined as $[15,17]$

$$
D(h)=d_{H}\{x \mid h(x)=h\},
$$

where $d_{H}$ is the Haussdorf dimension. The probability to observe an exponent $h$ at scale $a$ is then proportional to $a^{1-D(h)}$. Thus, the $\zeta(q)$ spectrum can be related to the singularity spectrum $D(h)$ by a Legendre transform, i.e., $\zeta(q)=\min _{h}[1+q h-D(h)]$. When $s(x)$ is monofractal, $h(x)$ is a constant $H$ independent of $x, D(H)=1$, and $\zeta(q)=q H$ is proportional to $q$. Conversely, if $s(x)$ is multifractal, $h(x)$ takes different values at different positions $x$, and $\zeta(q)$ is not proportional to $q$.

In practice, the values $\zeta(q)$ are obtained by fitting straight lines (when power law behavior is observed) on $\log -\log$ plots of $M_{q}$ versus scale $a$ for different moment orders $q$. The singularity spectrum $D(h)$ is then deducted from $\zeta(q)$. To verify if $\zeta(q)$ differs from a linear monofractal behavior, one needs to obtain $\zeta(q)$ for a large range of $q$ values and then proceed to fit the $\zeta(q)$ curve (for instance, $1 \leq q \leq 8$ in [6]; see also [19]).

While this has been the traditional way of estimating $\zeta(q)$, an alternate method introduced recently in the turbulence literature $[14,20]$, involves only a few straight line fits (as low as 3) while still accurately estimating the nonlinear behavior of the $\zeta(q)$ spectrum. To summarize this method, we start with the general expansion [14]:

$$
\ln M_{q}=\sum_{n=1}^{\infty} C_{n}(a) \frac{q^{n}}{n !},
$$

where $C_{n}(a)$ are the cumulants of $Q_{a} \equiv \ln |T[s](x, a)|$. One can demonstrate that the first two cumulants are the mean and standard deviation $Q_{a}$ :

$$
C_{1}(a)=\left\langle Q_{a}\right\rangle, \quad C_{2}(a)=\left\langle Q_{a}^{2}\right\rangle-\left\langle Q_{a}\right\rangle^{2} .
$$

Identifying the first derivative of Eq. (4) and of the logarithm of Eq. (1) with respect to $\ln (a)$, one finds

$$
\zeta(q)=c_{1} q+c_{2} q^{2} / 2 !+\cdots,
$$

where $c_{i} \equiv d C_{i}(a) / d \ln (a)$ are constants in the case of scale invariant signals. It turns out that the average value of the Hölder exponent is $\langle h\rangle=c_{1}$ and its variance $\left\langle h^{2}\right\rangle-$ $\langle h\rangle^{2}=-c_{2} / \ln (a)$. When the multiresolution coefficient $T[s](x, a)$ has Gaussian statistics (for example, when $s$ is a Brownian motion), $C_{2}(a)=\pi^{2} / 8$ and $c_{2}=0$.

The above developments imply that $\zeta(q)$ can be estimated from linear regressions of the cumulants $C_{n}(a)$ vs $\ln (a)$ [14]. For a monofractal signal, $c_{n}=0$, $\forall n \geq 2$ and only one linear regression is needed. For a multifractal signal, a quadratic $\zeta(q)$ approximation requires only two linear regressions. In comparison with the standard method based on the $q$ th order moments, the efficiency of the cumulant method becomes apparent.

If one simply wants a reliable estimate of the roughness exponent without questioning whether the signal is multifractal or not, it is best to measure $c_{1}$ rather than the commonly used exponent $\zeta(2)$. Indeed, any deviations from monofractality leading to a nonzero value of $c_{2}$ (either the signal is actually multifractal or is perturbed by experimental errors) will strongly influence the value of $\zeta(2)$. Note that a better estimator based on moment analysis would be $\zeta(1)$, proportionally less influenced by $c_{2}$ than $\zeta(2)$. In the following, we will illustrate these remarks looking at moments and cumulants obtained with the increments as multiresolution coefficients.

Influence of force and initial length. - In Fig. 2, we plot $\log _{10} M_{1}$ and $C_{1} / \ln (10)$ versus $\log _{10}\left(a / a_{0}\right)$ for five different couples of values $\left(F, L_{i}\right)$ during the fast growth stage. First, we see that near the discretization scale $a_{0}$ the slope is close to unity. This effect can be attributed to the
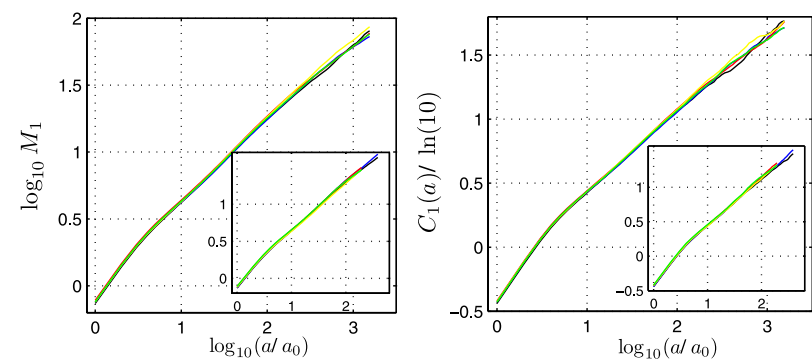

FIG. 2 (color online). (a) $\log _{10} M_{1}$ and (b) $C_{1} / \ln (10)$ versus scale calculated by the increments method for five different couples $\left(F, L_{i}\right)$ during fast (slow in inset) crack growth. 
discreteness of the signal [21]. In the following, we will concentrate on larger scales $a>4 a_{0}(\simeq$ maximum fiber diameter) where this effect can be neglected. Second, we see no dependence of $M_{1}$ and $C_{1}$ on the force or the initial crack size. The same independence is observed for the slow growth stage. Thus, we can average the moments or cumulants over all the 102 crack fronts whatever are the forces or initial crack lengths. For each front, we also compute the derivative of the moments or cumulants and average over all the fronts to get the mean local slope and statistical error of the mean as a function of scale. The large number of crack fronts allows us to greatly improve the precision of the statistical analysis.

First and second order moments. - Figures 3(a) and 3(b) show log-log plots of $M_{1}$ and $M_{2}$ versus $a / a_{0}$ for slow and fast growth, and Figs. 3(c) and 3(d) the corresponding mean local slopes $m_{1}(a)$ and $m_{2}(a)$. Whatever are the exact scaling properties of $M_{1}$ and $M_{2}$, a systematic difference, larger than the error bars, between the slopes of the two growth regimes is observed in a wide range of scales. Since $M_{1}$ and $M_{2}$ are not perfect straight lines, we can question the existence of true scaling laws. Assuming scaling laws do exist would mean that the signal is not monofractal since $\left\langle m_{1}\right\rangle \neq\left\langle m_{2} / 2\right\rangle$. In the present case, it is better to estimate the roughness exponent by looking at the first order cumulant, which is by construction insensitive to deviations from monofractality.

First and second order cumulants. - In Figs. 4(a) and 4(b), we plot $C_{1}(a)$ and $C_{2}(a)$ divided by $\ln (10)$ versus $\log _{10}\left(a / a_{0}\right)$ for the two growth regimes. For later use, the mean local slope of $C_{1}(a)$ [Fig. 4(c)] will be noted $c_{1}^{F}(a)$ for the fast regime, $c_{1}^{S}(a)$ for the slow one, and $\Delta c_{1}(a)=$ $c_{1}^{S}(a)-c_{1}^{F}(a)$ the slope difference. There is a clear difference between slow and fast crack growth [Fig. 4(c)] and
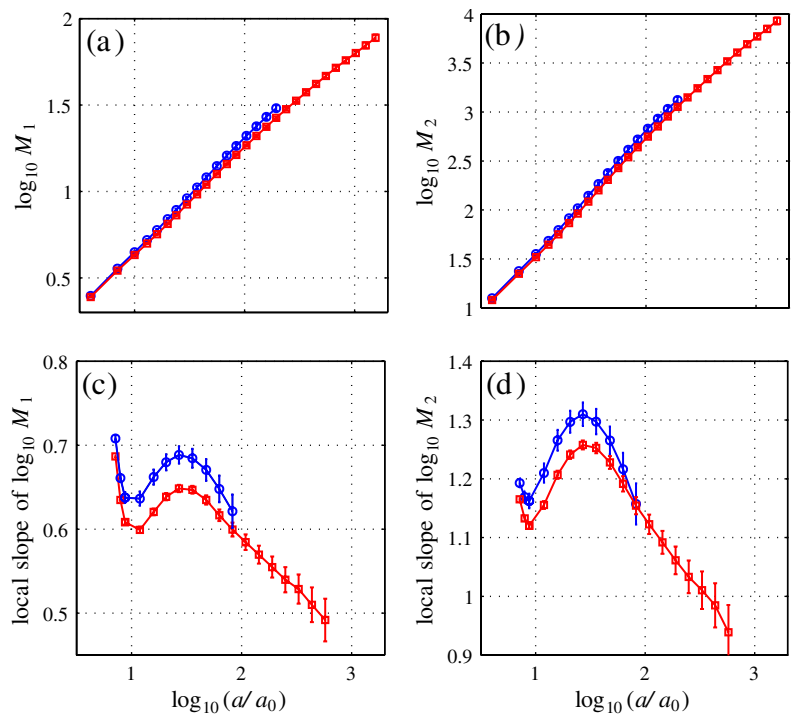

FIG. 3 (color online). (a),(b): $M_{1}$ and $M_{2}$ for slow (circles) and fast (squares) crack growth; (c),(d): Corresponding local slope. The error bars are determined as statistical errors of the mean ( $\equiv$ standard deviation $/ \sqrt{N}$ with $N=102$ ). much less overlap between the values of $c_{1}^{F}(a)$ and $c_{1}^{S}(a)$ than for $m_{1}$ or $m_{2}$. As for $M_{1}$ or $M_{2}, C_{1}(a)$ is not perfectly linear and, in a certain range of scales $\left(10<a / a_{0}<10^{2}\right)$, $C_{2}$ is close to the theoretical value for a signal with Gaussian statistics [22]. The local extremum observed in both regimes for $a / a_{0} \simeq 10^{1.5}(a \simeq 500 \mu \mathrm{m})$ in Fig. 4(c), $3(\mathrm{c})$ and 3(d) might correspond to a characteristic scale of the material. Indeed, this scale is of the same order than the fiber length in paper.

Scaling laws. -From the various plots, we can already conclude that it is not so easy to find a range of scales for which true scaling laws are observed. For $a / a_{0} \leqslant 10$, the slope of $C_{1}(a)$ is changing a lot because we start to feel the discretization effect previously discussed [21]. We clearly see that for $a / a_{0} \gtrsim 10^{2}$, the slope of $C_{1}(a)$ is again changing significantly and seems to go towards 0.5 . Thus, if a scaling law exists, it is observed mainly at intermediate scales where the slope values are the most stable. The same conclusion can be reached by looking at $C_{2}(a)$. At large scale, $C_{2}(a)$ decreases to values lacking physical meaning since they become smaller than the Gaussian value and is very sensitive to discretization effects at small scales. Trying to estimate the slope of $C_{2}(a)$ at intermediate scales $\left(10<a / a_{0}<10^{2}\right)$, one finds that $c_{2}$ is very close to zero for the fast crack growth and between -0.08 and -0.02 for the slow part. However, given the large error bars [see Fig. 4(d)], we can neither conclude that there is a difference between the two growth regimes for $c_{2}$, nor that there is a mono- or multifractal behavior. For that reason, we will focus only on the first order cumulant to estimate the roughness exponent.

Roughness exponent. - In the range of scales where the scaling is reasonably good $\left(10<a / a_{0}<10^{2}\right)$, the rough-
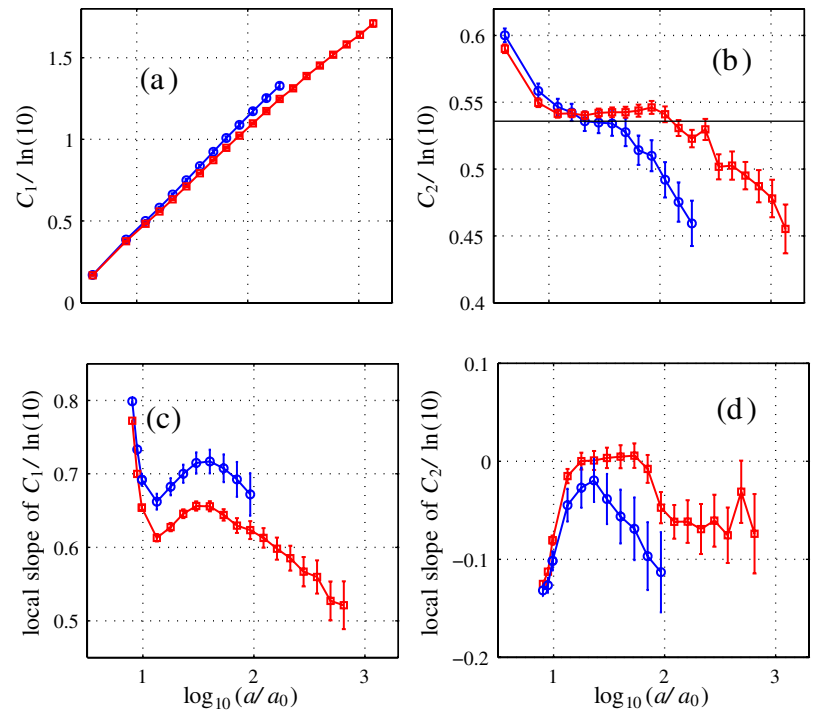

FIG. 4 (color online). (a),(b): $C_{1}$ and $C_{2}$ versus scale with statistical error bars. In (b), the horizontal solid line corresponds to a signal with Gaussian statistics. (c),(d): Corresponding local slope of cumulants. Error bars give the statistical error of the mean. 
TABLE I. Roughness exponents $c_{1}(=\langle h\rangle)$ for fast and slow crack growth, and their difference using various methods.

\begin{tabular}{lccc}
\hline \hline Method & Fast growth & Slow growth & Difference \\
\hline SF & $0.64 \pm 0.02$ & $0.70 \pm 0.02$ & $0.06 \pm 0.01$ \\
CWT & $0.65 \pm 0.02$ & $0.73 \pm 0.02$ & $0.07 \pm 0.01$ \\
WTMM & $0.64 \pm 0.01$ & $0.70 \pm 0.02$ & $0.06 \pm 0.01$ \\
\hline \hline
\end{tabular}

ness exponents $c_{1}^{F}$ and $c_{1}^{S}$, as well as their errors, are computed as the mean and standard deviation of $c_{1}^{F}(a)$ or $c_{1}^{S}(a)$. The error on $c_{1}^{F}$ or $c_{1}^{S}$ is indeed dominated by the variations across scale not by the statistical errors [Fig. 4(c)]. In the same scale range, we also compute the mean difference $\Delta c_{1}$. The variations of $\Delta c_{1}(a)$ across scale are very small and the error on $\Delta c_{1}$ is then dominated by the statistical errors [Fig. 4(c)]. Thus, the error on $\Delta c_{1}$ will be significantly smaller than the ones on $c_{1}^{F}$ and $c_{1}^{S}$. We show in Table I the measured exponents and their difference as well as the corresponding errors using various methods [structure functions (SF), continuous wavelet transform (CWT) [15], and wavelet transform modulus maxima (WTMM) [16] using the 1st derivative of Gaussian]. They all give a difference between the two growth regimes. For instance, SF give a difference of $0.06 \pm 0.01$ with a roughness exponent of $0.64 \pm 0.02$ for the fast regime and $0.70 \pm 0.02$ for the slow regime [23].

Conclusion. - Fracture fronts in paper do not follow true scaling laws, either mono- or multifractal. However, we find a drop in the local roughness when the crack goes from subcritical to fast growth. In the subcritical regime, the physical mechanism for crack growth is thermal activation [12], while in the fast growth regime, the system is mechanically unstable. Dynamical instabilities during fast crack growth were shown to decrease the roughness [9] which could explain our observations. In the slow regime, roughness is probably controlled more by the material disorder than by dynamical effects. There is a characteristic scale $a \simeq 500 \mu \mathrm{m}$, close to the fiber length, which could come from fiber pullout rather than breakage. Easier pullout in the slow growth regime, compared to the fast growth regime, might also play a role in the roughness difference.

We thank S. Ciliberto for fruitful discussions. This work was funded with Grant No. ANR-05-JCJC-0121-01.

*Present address: Fysisk Institutt, Universitetet i Oslo, PostBoks 1048 Blindern, 0316 Oslo, Norway.

†Loic.Vanel@ens-lyon.fr
[1] B. B. Mandelbrot, D. E. Passoja, and A. J. Paullay, Nature (London) 308, 721 (1984); A.L. Barabási and H.E. Stanley, Fractal Concepts in Surface Growth (Cambridge University Press, Cambridge, England, 1995).

[2] E. Bouchaud, G. Lapasset, and J. Planès, Europhys. Lett. 13, 73 (1990); E. Bouchaud, J. Phys. Condens. Matter 9, 4319 (1997); K. J. Måløy, A. Hansen, E. L. Hinrichsen, and S. Roux, Phys. Rev. Lett. 68, 213 (1992).

[3] E. Bouchbinder, I. Procaccia, and S. Sela, Phys. Rev. Lett. 95, 255503 (2005); L. Ponson, D. Bonamy, and E. Bouchaud, Phys. Rev. Lett. 96, 035506 (2006).

[4] I. L. Menezes-Sobrinho, M. S. Couto, and I. R. B. Ribeiro, Phys. Rev. E 71, 066121 (2005); A.S. Balankin, O. Susarrey, and J.M. González, Phys. Rev. Lett. 90, 096101 (2003).

[5] J. M. Lopez, M. A. Rodriguez, and R. Cuerno, Phys. Rev. E 56, 3993 (1997).

[6] E. Bouchbinder, I. Procaccia, S. Santucci, and L. Vanel, Phys. Rev. Lett. 96, 055509 (2006).

[7] J. Schmittbuhl and K. J. Måløy, Phys. Rev. Lett. 78, 3888 (1997); P. Daguier, B. Nghiem, E. Bouchaud, and F. Creuzet, Phys. Rev. Lett. 78, 1062 (1997); J. M. Boffa et al., Eur. Phys. J. B 7, 179 (1999).

[8] A.S. Balankin, O. Susarrey, and A. Bravo, Phys. Rev. E 64, 066131 (2001).

[9] J.-F. Boudet, S. Ciliberto, and V. Steinberg, J. Phys. II 6, 1493 (1996).

[10] M. J. Alava, P. K. V. V. Nukala, and S. Zapperi, Adv. Phys. 55, 349 (2006).

[11] V. Horvath, J. Kertész, and F. Weber, Fractals 1, 67 (1993); L. I. Salminen, M. J. Alava, and K. J. Niskanen, Eur. Phys. J. B 32, 369 (2003).

[12] S. Santucci, L. Vanel, and S. Ciliberto, Phys. Rev. Lett. 93, 095505 (2004); S. Santucci et al., Europhys. Lett. 74, 595 (2006).

[13] J. Schmittbuhl, J.-P. Vilotte, and S. Roux, Phys. Rev. E 51, 131 (1995).

[14] J. Delour, J. F. Muzy, and A. Arneodo, Eur. Phys. J. B 23, 243 (2001)

[15] G. Parisi and U. Frisch, in Turbulence and Predictability in Geophysical Fluid Dynamics, edited by M. Ghil, R. Benzi, and G. Parisi (North-Holland, Amsterdam, 1985), p. 84.

[16] J.F. Muzy, E. Bacry, and A. Arneodo, Int. J. Bifurcation Chaos Appl. Sci. Eng. 4, 245 (1994).

[17] S. Mallat, A Wavelet Tour of Signal Processing (Academic, Boston, 1997).

[18] I. Simonsen, A. Hansen, and O. M. Nes, Phys. Rev. E 58, 2779 (1998).

[19] A. L. Barabási et al., Phys. Rev. A 45, R6951 (1992).

[20] L. Chevillard, S. G. Roux, and E. Leveque et al., Phys. Rev. Lett. 91, 214502 (2003); 95, 064501 (2005).

[21] S. J. Mitchell, Phys. Rev. E 72, 065103(R) (2005).

[22] S. Santucci et al., Phys. Rev. E 75, 016104 (2007).

[23] These values are consistent with the literature $[4,11]$. 